\title{
Erratum to: Static and Dynamic Green's Functions in Peridynamics
}

\author{
Linjuan Wang ${ }^{1} \cdot$ Jifeng $\mathrm{Xu}^{2} \cdot$ Jianxiang Wang ${ }^{1,3}$
}

Published online: 10 October 2016

(C) Springer Science+Business Media Dordrecht 2016

\section{Erratum to: J Elast (2016)}

\section{DOI 10.1007/s10659-016-9583-4}

1. The first sentence of the Introduction should be changed to: During the past several decades, it has been recognized that "neither distributed damage in materials nor transitions to discrete microstructural models can be adequately characterized by local constitutive relations between stress and strain tensors" [1].

2. $\mu$ and $\frac{2 \mu}{1-v}$ in the sentence under Equation (67) should be changed to $\mu k^{2}$ and $\frac{2 \mu}{1-v} k^{2}$, respectively.

3. $\mu$ and $\frac{2 \mu(1-v)}{1-2 v}$ in the sentence under Equation (96) should be changed to $\mu k^{2}$ and $\frac{2 \mu(1-v)}{1-2 v} k^{2}$, respectively.

4. Equation (103) should be modified as

$$
u_{i}^{(j)}=\frac{P}{h_{0}} \cdot \frac{(1+v)}{8 \pi \mu}\left(-\frac{3-v}{1+v} \delta_{i j} \ln x+\frac{x_{i} x_{j}}{x^{2}}\right) .
$$

The online version of the original article can be found under doi:10.1007/s10659-016-9583-4.

\section{J. Wang}

jxwang@pku.edu.cn

1 State Key Laboratory for Turbulence and Complex Systems, Department of Mechanics and Engineering Science, College of Engineering, Peking University, Beijing 100871, P.R. China

2 Beijing Aeronautical Science and Technology Research Institute, Beijing 100083, P.R. China

3 CAPT, HEDPS and IFSA Collaborative Innovation Center of MoE, Peking University, Beijing 100871, P.R. China 